\title{
"Los rincones, los talleres y los proyectos como recursos para trabajar la sostenibilidad en educación infantil"
}

\section{"corners, workshops and projects as resources to work on sustainability in early childhood education"}

DOI: $10.46932 / \mathrm{sfjdv2n5-137}$

Received in: Oct 1st, 2021

Accepted in: Dec 30th, 2021

\author{
Sofía Alacid de Pascual \\ Graduada en Educación Infantil \\ Universidad de Murcia \\ Calle Gabriel García Márquez 2 \\ E-mail: investigacionrgformacion@gmail.com
}

\begin{abstract}
RESUMEN
El objetivo de este artículo es la difusión de la educación para el desarrollo sostenible en la etapa de Educación Infantil, aportando un proyecto educativo que toma como marco de referencia las problemáticas ambientales relacionadas con la contaminación del mar. En él se combinan las propuestas realizadas por la UNESCO, que buscan cambiar los estilos, hábitos y valores de la sociedad, incorporando la educación para el desarrollo sostenible en la práctica docente. El aprendizaje por rincones, junto con el trabajo cooperativo y los talleres se abarcan dentro del proyecto de trabajo, y se trata de metodologías que permiten a los estudiantes interactuar y conectar con los problemas de la comunidad, generando oportunidades de aprendizaje significativo. Además, este proyecto permite incorporar recursos a los docentes sobre esta temática y hacer reflexionar a los alumnos y a su entorno social, sobre la importancia de cuidar el mar, que es un bien y una responsabilidad de toda la población.
\end{abstract}

Palabras clave: Desarrollo sostenible, Educación Infantil, Rincones de trabajo, Trabajo cooperativo, talleres, Proyecto de trabajo.

\begin{abstract}
The aim of this article is to disseminate education for sustainable development in the Early Childhood Education stage, providing an educational project that takes as a frame of reference the environmental problems related to sea pollution. It combines the proposals made by UNESCO, which seek to change the styles, habits and values of society, incorporating education for sustainable development in teaching practice. Learning by corners, along with cooperative work and workshops are included in the work project, and these are methodologies that allow students to interact and connect with the problems of the community, generating opportunities for meaningful learning. In addition, this project allows teachers to incorporate resources on this topic and to make students and their social environment reflect on the importance of caring for the sea, which is an asset and a responsibility of the entire population.
\end{abstract}

Keywords: Sustainable development, Early Childhood Education, Work corners, Cooperative work, workshops, Work project. 


\section{INTRODUCCIÓN}

La educación para la sostenibilidad se ha convertido en una necesidad de la sociedad actual, ya que el cambio climático ha propiciado una mayor concientización sobre los problemas ambientales y la elaboración de múltiples guías para ayudar al profesorado a desarrollar su labor docente en este ámbito. Además, diferentes sucesos que han trascendido recientemente a la opinión pública han aumentado la preocupación por generar una mayor conexión, entre la temática medioambiental y el día a día de los centros educativos.

Por ello, el objetivo de este artículo es orientar a los docentes de educación Infantil sobre cómo abordar de manera concreta la educación para la sostenibilidad, por medio de las metodología habituales utilizadas actualmente en las aulas de infantil (trabajo por proyectos, rincones y talleres), de manera que se pueda abordar la educación para el desarrollo sostenible, no sólo de forma transversal y globalizada, sino también a través de actividades concretas adecuadas para estas edades como, proyectos de trabajo, talleres o rincones.

En primer lugar, para plantear talleres, proyectos o rincones, con los que trabajemos de forma explícita la educación para la sostenibilidad, es necesario conocer en profundidad cada una de estas metodologías, su definición, características y aspectos más relevantes a tener en cuenta, ya que esto nos facilitará posteriormente realizar propuestas concretas para trabajar la educación para el desarrollo sostenible con cada una de las metodologías.

Las propuestas de trabajo que se realizan en este artículo pretender dar ejemplo de cómo abordar este tipo de enseñanza en las aulas de infantil, ayudar así de alguna manera a los docentes en su tarea educativa, ya que la formación del profesorado actual sobre este tipo de educación es escasa, al igual que el material u orientaciones concretas para esta etapa educativa, ya que en su mayoría, las experiencias dirigidas a fomentar valores para la sostenibilidad van dirigidas a las etapas educativas de educación primaria o secundaria, debido a la poca relevancia que socialmente se le otorga a la educación infantil.

Sin embargo, es en esta etapa donde se desarrollan las bases o capacidades que permitirán al alumnado, en las siguientes etapas educativas, alcanzar las competencias correspondientes a su etapa educativa, y, ya puesto que lograr sociedades más sostenibles, requiere ciudadanos competentemente sostenibles, es de vital importancia educar para la sostenibilidad desde edades tempranas.

\section{LOS PROYECCTOS DE TRABAJO}

Establecer el tipo de metodología con el que se va a trabajar en el aula, supone en ocasiones, sobre todo los primeros años de docencia, un reto para los maestros. En educación infantil concretamente, las metodologías que utilicemos deben de caracterizarse por ser abiertas, flexibles, que atiendan al principio de globalización de la etapa, es decir, no trabajar las áreas curriculares de manera fragmentada sino 
abarcarlas, al igual que la perspectiva de los discentes de esta etapa, de forma integral, que fomenten el aprendizaje significativo...

Una de las metodologías actualmente más reconocidas en educación infantil son los proyectos de trabajo. Se trata de una manera distinta a la convencional, de entender el proceso de enseñanzaaprendizaje, que se organiza en torno a una temática determinada y, además, permite atender a la diversidad del alumnado, ya que se desarrolla en un entorno de interacción, investigación y actividad (Domínguez,2003).

El origen del trabajo por proyectos se sitúa en Dewey y, principalmente en Kilpatrick, el cual los dio a conocer como una propuesta que fomenta en los alumnos la iniciativa personal, la interacción la solidaridad y el ejercicio de la libertad responsable, así como promoviendo en los alumnos un compromiso real con el aprendizaje. Se estructuran en actividades organizadas en torno a una finalidad concreta y un tema motivador que despierte el interés del alumnado, además de tratar los contenidos de forma globalizada. Actualmente, el modelo de trabajo por proyectos adoptado en las escuelas de infantil sienta sus bases en las propuestas pedagógicas de las Escuela Reggio Emilia, la cual parte de la observación y documentación de la actividad del niño (García, 2013).

Según Miralles y Rivero (2012) los proyectos son "una metodología ligada a la perspectiva constructivista del aprendizaje a las tareas del aprendizaje por descubrimientos, utilizando estrategias de indagación que combina diferentes propuestas de actividad complementarias entre sí para la enseñanza de un tema concreto". Trabajar por proyectos supone materializar el planteamiento metodológico y pedagógico de los docentes, consiguiendo que los niños exploren y experimenten en función de sus propios intereses (García, 2013).

Según Vizcaíno (2008) los proyectos de trabajo se conforman en 4 fases que, kilpatrick estableción, y serán las mismas fases que compongan el proyecto que relizaremoos en elapartaado 4:

1. Inicio. Esta fase es una de las más importantes del proyecto, la maestra descubrirá las ideas previas de los alumnos, en relación al centro de interés que se trate y partiendo de ellas, realizará las adaptaciones necesarias de su planificación docente a las necesidades de aprendizaje de los alumnos, esto ayudará a que los alumnos consigan un aprendizaje significativo que parta de sus ideas previas, y se enriquezca gracias a la confrontación de lo que sabían, con los nuevos contenidos aprendidos.

2. Recogida de Información. Introducción de la nueva información. En esta fase se tratarán por primera vez los nuevos contenidos, de forma que el alumno vaya descubriendo poco a poco lo que queremos que aprenda, mediante la recogida de información. 
3. Desarrollo y aplicación de los contenidos. En esta fase se trabajarán los contenidos e información recogida en la fase anterior, mediante tareas activas que requieren poner en práctica la información recogida y su aplicación.

4. Cierre, reflexión y conclusiones. La última de las fases trata de concluir el proyecto haciendo una reflexión de todo lo que se ha aprendido, y valorando los contenidos trabajados.

Por su parte, Beresaluce (2009) señala que los proyectos de trabajo fomentan especialmente las relaciones interpersonales y el trabajo cooperativo.

\subsection{EL TRABAJO COOPERATIVO}

El trabajo cooperativo es no es solo una modalidad de trabajo, sino que también conforma una metodología complementaria al trabajo por Proyectos, dentro de los mismos podemos incluir actividades de carácter cooperativo.

Además, el trabajo cooperativo no solo cumple con uno de los principios metodológicos más relevantes de la práctica educativa; ya que, tiene como finalidad principal hacer al alumno participe de su proceso de aprendizaje y convertirlo en un agente activo, saliendo del individualismo tradicional que ha impregnado las aulas durante años, sino que también, es adecuado para la educación para la sostenibilidad porque la colaboración y cooperación de los ciudadanos es un rasgo distintivo de la educación para el desarrollo sostenible.

Johnson, Johnson y Holubec, (1999) definen el aprendizaje cooperativo como el proceso en el que: "los estudiantes trabajan juntos para lograr objetivos comunes, asegurándose de que ellos mismos y sus compañeros de grupo completen la tarea de aprendizaje asignada". A la hora de plantear actividades de trabajo cooperativo se deberán tener en cuenta las siguientes características básicas del mismo (Johnson et al, 1999):

- La interdependencia positiva, que hace que los alumnos se comprometan conseguir un objetivo junto.

- La responsabilidad individual y grupal, que se da cuando el alumno procura que su trabajo y, el de su grupo llegue a buen término.

- La interacción estimuladora, prioritariamente cara a cara: Esta interacción es la que promueve que los miembros se apoyen unos a otros y ejerzan estímulos positivos mediante felicitaciones de los compañeros o bien, compartiendo materiales y ayudándose unos a otros.

- Las prácticas interpersonales y grupales, imprescindibles ya que en el trabajo cooperativo además de aprender sobre los contenidos de las tareas han de aprender a trabajar en equipo. 
- La evaluación grupal, que posibilita la valoración del trabajo por parte de los alumnos, una vez finalizado el mismo, analizando los puntos fuertes y los puntos débiles de la ejecución de la tarea. Para trabajar con esta metodología en un aula concreta es necesario que la maestra realice una adaptación de la metodología al aula en la que se va a poner en práctica. Para ello, una vez que la maestra organice y distribuya los grupos, nombrará los roles que cada alumno debe desempeñar en su grupo y les dará una pegatina que les identifique con el rol concreto, bien sea:

Encargado del silencio, que tendrá que procurar que su grupo no levante mucho la voz.

Encargado del material, que deberá de proporcionar al grupo el material que necesite.

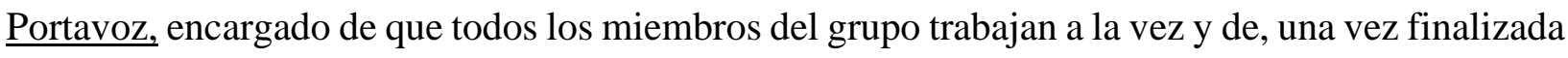
la tarea, explicar delante de toda la clase los puntos fuertes y débiles, que anteriormente habrá acordado con el resto del grupo.

Con respecto a la exposición del trabajo a toda la clase, la maestra irá dirigiendo la exposición mediante preguntas al grupo, actuando como guía, para ayudarlos a reflexionar sobre los puntos fuertes y débiles de su trabajo.

\subsection{LOS CUATRO PILARES DE LA EDUACIÓN}

Para la planificación docente me basaré en los cuatro pilares de la educación (Delors, 1996):

- Aprender a conocer.

- Aprender a hacer.

- Aprender a vivir juntos.

- Aprender a ser.

Siguiendo estos cuatro pilares podremos guiar al alumno hacia una educación integradora y globalizada que, a su vez, cumple con los principios metodológicos de una enseñanza activa en la que el alumno forma parte del proceso construyendo su aprendizaje. Se favorece el aprendizaje significativo, es decir, un aprendizaje para la vida, que el alumno pueda utilizar en otras situaciones y contextos diferentes al ámbito escolar.

\section{TALLERES}

El aprendizaje según Candelo, Ortiz y Unger (2003) significa “adquirir información, comprenderla, memorizarla y llevarla a la práctica” (p. 13). Además, actualmente, más que nunca y, en especial para este proyecto, aprender tiene como finalidad principal capacitar.

Con el aprendizaje podemos facilitar cambios positivos en el ser humano, y los maestros como facilitadores de este tenemos la responsabilidad de escoger las mejores herramientas para favorecer dichos cambios. Por ello, he incluido la metodología de talleres en la propuesta de actividades, pues estos 
coinciden con el requisito, finalidad o propósito de este proyecto, capacitar a nuestro alumnado para favorecer el desarrollo sostenible en la zona del Mar Menor, de este modo también los talleres nos son de gran ayuda para conseguir el objetivo principal de este trabajo.

Pero ¿qué son exactamente los talleres?, Careaga, Cirillo, y Da Luz, S (2006) afirman que los talleres implican;

"Un lugar donde se trabaja y se elabora. Es una forma de enseñar y aprender mediante la realización de algo. Se aprende desde lo vivencial y no desde la transmisión. Predomina el aprendizaje sobre la enseñanza. Se trata entonces de un aprender haciendo, donde los conocimientos se adquieren a través de una práctica concreta, realizando algo relacionado con la formación que se pretende proporcionar a los participantes. Es una metodología participativa en la que se enseña y se aprende a través de una tarea conjunta" (p.5).

Los talleres, por tanto, nos facilitarán actividades de investigación con los alumnos, ya que, según esta metodología, ellos deberán ejercer el papel activo, el papel de investigador. Además, los talleres favorecen tres aspectos que son de total relevancia para llevar a cabo nuestro proyecto; la atención a necesidades, tratando una problemática perteneciente al entorno de nuestro alumnado; la participación, la cual es un elemento importante para fomentar la motivación y la capacitación y; la visualización, es decir, visualizando el problema se darán soluciones para el mismo, con las que el aprendizaje culmina (Candelo, et al. 2003).

Realizar grupos de trabajo para la realización de los talleres es un requisito indispensable para trabajar con esta metodología, los grupos serán reducidos, de tres personas, por ejemplo. Aun conociendo la definición de la metodología basada en talleres, es conveniente que para llevar a la práctica esta estrategia conozcamos claramente las principales características de un taller, según Carega et al. (2006) estas son:

- "Se basa en la experiencia de los participantes.

- Es una experiencia integradora donde se unen la educación y la vida, los procesos intelectuales y afectivos.

- Está centrado en los problemas e intereses comunes del grupo.

- Implica una participación activa de los integrantes.

- Puede utilizar diversas técnicas, especialmente la discusión en grupo" (p.6).

Además, a la hora de diseñar un taller el maestro debe tener en cuenta las características del alumnado para realizar un taller personalizado, que tenga en cuenta el ritmo de aprendizaje del grupoclase. 


\section{RINCONES}

Los rincones de actividad o trabajo permiten integrar actividades de aprendizaje de distintas áreas en un solo ambiente, siendo esta una de las características principales de esta metodología (Laguía y Vidal, 2000):

"Trabajar por rincones quiere decir organizar la clase en pequeños grupos que efectúan simultáneamente actividades diferentes" (p.18)

Partiendo de esta definición principal, su puesta en práctica puede variar debido a la adaptación que la maestra propone para su aula teniendo en cuenta su realidad contextual y sus necesidades. En la puesta en práctica de esta metodología del trabajo por rincones, se ha decidido optar por las rotaciones, es decir, los alumnos irán pasando por los rincones en turnos y con los grupos de trabajo establecidos previamente, con el fin de conseguir que todos los alumnos pasen por todos los rincones. Cada grupo de trabajo estará compuesto por seis alumnos identificándose con números del uno al cuatro.

Todas las sesiones que se han diseñado según esta metodología se estructuran en tres partes:

1. Mini-lección. La maestra explicará las actividades que se van a realizar en cada rincón y seguidamente asignará a cada grupo un rincón.

2. Acción. Los alumnos deberán realizar la actividad de su rincón y, una vez que finalicen, podrán jugar libremente en su lugar hasta que todos terminen las actividades asignadas.

3. Rotación. Los grupos recogerán los materiales con los que estaban trabajando y pasarán a realizar la actividad del siguiente rincón, de esta manera conseguiremos que todos los alumnos realicen las actividades diseñadas para cada rincón y, por ende, trabajarán todos los contenidos propuestos para esa sesión.

Conviene destacar que esta metodología se lleva a cabo en dos sesiones concretas, en las cuales, se cuenta con la presencia de padres que voluntariamente quieran y puedan participar. Se le asignará a cada padre o madre uno de los rincones para que actúen como agente de intervención y mediador del diálogo en el grupo de trabajo. En el presente proyecto se considera clave la participación de los padres como un elemento que enriquece el contexto del proceso enseñanza-aprendizaje, y además, debido a la simultaneidad de tareas, la presencia de los padres en este tipo de sesiones se convierte en un apoyo relevante y atractivo para los alumnos (Flecha, 2006).

\section{PROYECTO “CUIDAMOS DEL MAR" A TRAVÉS DE TRABAJO COOPERATIVO, TALLERES Y RINCONES}

Es necesario destacar que las actividades propuestas a continuación, van dirigidas a un alumnado perteneciente a un aula de 5 años, pero aun así, esta batería de actividades puede adaptarse a cualquier nivel de esta etapa educativa. 
FASE 1: INICIO. MOTIVACIÓN, DETECCIÓN DE IDEAS PREVIAS.

Actividad de motivación: "los peces del mar nos piden ayuda"

\begin{tabular}{|c|l|}
\hline Objetivo & Contenido \\
\hline $\begin{array}{c}\text { Mostrar interés y motivación por explorar el } \\
\text { aula y por comenzar el nuevo proyecto, conocer las } \\
\text { ideas previas de los alumnos. }\end{array}$ & El mar contaminado. \\
\hline
\end{tabular}

Temporalización: 30 minutos.

Descripción de la actividad:

En esta sesión la maestra motivará a los alumnos mediante la preparación de la clase, para ello colocará por el aula, sobre todo por la zona de la asamblea, elementos del mar como conchas, algas, dibujos de peces, estrellas de mar y en las ventanas pegará papel celofán azul y tiras de papel verde colgando del techo del aula, que simularán las algas del mar. En medio de la asamblea colocará una botella de cristal que llevará dentro una carta que la maestra leerá.

La actividad se realizará después del patio, pues dará lugar a que los niños salgan de la clase y la maestra aprovechará para preparar la clase durante el recreo, para que cuando vuelvan ya esté todo listo y sea un elemento sorpresa e inesperado que motive a los alumnos, durante diez minutos el alumnado se dedicará a explorar la clase libremente, con la excepción de que no podrán tocar la botella que hay en medio de la asamblea.

Una vez hayan pasado los primeros diez minutos de la sesión, la maestra indicará que es el momento de sentarse en la asamblea formando un círculo, la maestra se sentará con los alumnos y dirá:

- ¿Qué creéis que puede haber en esa botella?

- (Dejar tiempo para las intervenciones de los niños)

- ¿Queréis que lo descubramos?

A continuación, la maestra pedirá al encargado de ese día que saque lo que hay dentro de la botella y se lo entregue, seguidamente la maestra se dispondrá a mirar lo que es y dirá:

- ¡Es una carta! Aquí pone que nos la ha mandado un caballito de mar. Vamos a ver que nos cuenta el caballito de mar.

Cuando la maestra haya leído la carta, pasará a mantener un diálogo con los alumnos, y realizará las siguientes preguntas sobre el Mar.

- ¿Qué os ha parecido esta carta?

- ¿Conocéis algún Mar? ¿Habéis estado alguna vez en alguna playa?

- ¿Habéis visto alguna vez un caballito de mar?

- ¿Cómo son los caballitos de mar?

- ¿Estaba limpia el agua del mar? 
Una vez acabado el diálogo se dará por comenzado el proyecto, la maestra como en todos los proyectos, invitará a los niños y a los padres a que traigan recursos, materiales o información sobre el Mar y sus animales, y se inaugurará el rincón del Mar, dejando su carta en un rincón que la maestra habrá preparado.

\section{FASE 2. DESARROLLO DE LOS CONTENIDOS}

\begin{tabular}{|c|c|}
\hline Objetivo específico: & Contenido específico: \\
\hline $\begin{array}{l}\text { Reflexionar sobre la importancia del agua } \\
\text { para nosotros y para los demás seres vivos, } \\
\text { especialmente para el caballito de mar. }\end{array}$ & $\begin{array}{l}\text { Usos las personas hacen del agua y usos que } \\
\text { los seres vivos del Mar, como el caballito de mar, } \\
\text { hacen del agua. }\end{array}$ \\
\hline
\end{tabular}

Temporalización: 40 minutos.

Metodología: Trabajo cooperativo.

Descripción de la actividad:

Esta actividad se realizará en la asamblea y consistirá en hacer entre todos, con la ayuda de la maestra, una reflexión sobre los usos que hacemos del agua, para ello la maestra se sentará en la asamblea con los alumnos, que estarán todos colocados formando un círculo.

Una vez estén todos sentados la maestra lanzará una pregunta: ¿Para que usamos el agua?, seguidamente la maestra responderá 'Yo uso el agua para bebérmela y para hacer macarrones, ¿y tú?' y mientras pregunta 'y tú' le pasará la pelota a un niño y este niño tendrá que decir para que usa el agua, y preguntárselo a otro compañero, del mismo modo que la maestra, esto se realizará consecutivamente hasta que todos los niños hayan hecho su aportación. Después la maestra dará algunas ideas más sobre el uso que hacemos del agua y seguidamente lanzará la siguiente pregunta, ¿CCreéis que podríamos vivir sin agua?', dando lugar a un pequeño debate, en el que los alumnos deben de razonar su respuesta ya sea afirmativa o negativa.

Después la maestra colocará en medio de la asamblea varias imágenes, una persona, un pez, una planta, un caballito de mar y una almeja. La maestra les pedirá a los alumnos que miren esas imágenes durante un minuto, inmediatamente les preguntará 'De todo lo que hay aquí ¿hay algo que no necesite agua para vivir?', los alumnos deberán de dar una respuesta razonada, y al ver que todos necesitan agua para vivir, la maestra reflexionará con los alumnos sobre la importancia del agua, aclarando que no solo nosotros necesitamos agua para vivir sino que también la necesitan plantas y animales, sobre todo los que viven en el mar como el caballito de mar.

Seguidamente tocaría la parte práctica de la sesión, que consistiría en que los alumnos en sus grupos de trabajo cooperativo dibujaran, en un folio con forma de circulo, algún uso que hicieran del agua ellos o algún animal, conforme vayan acaban sus dibujos con la aplicación de Realidad Aumentada 'Quiver' de la Tablet de la clase, irían grupo por grupo enfocando el dibujo de su círculo, y verían como 
el circulo a través de la cámara de la Tablet se convierte en una esfera que va cambiando de movimiento y formas, mientras los alumnos usan la realidad Aumentada, la maestra les preguntaría: ‘¿no se parece vuestra esfera al planeta tierra?, el agua es tan importante que todo el mundo la necesita'.

RINCONES "El caballito de mar"

\begin{tabular}{|c|c|}
\hline Objetivo específico: & $\begin{array}{l}\text { Contenido específico: } \\
\end{array}$ \\
\hline $\begin{array}{l}\text { Aprender las características principales que } \\
\text { más identifican al caballito de mar por medio de } \\
\text { actividades globalizadas que incluyen la seriación } \\
\text { numérica del } 1 \text { al } 30 \text {, la lectoescritura del nombre } \\
\text { 'Caballito de mar', la expresión plástica del caballito } \\
\text { de mar en su casa y el desarrollo de la habilidad } \\
\text { espacial. }\end{array}$ & $\begin{array}{l}\text { La seriación numérica, escritura del nombre } \\
\text { caballito de mar, el mar y las partes del caballito de } \\
\text { mar }\end{array}$ \\
\hline
\end{tabular}

Temporalización: El tiempo necesario para realizar estas actividades son 2 horas en total, una antes del patio y otra después del patio, en cada hora los grupos rotaran dos veces de rincón

Metodología: Rincones de trabajo.

Descripción de la actividad:

Esta sesión se divide en tres, una primera parte explicativa que se realizará en la asamblea y consistirá en la explicación que dará la maestra sobre las actividades que se realizan en cada rincón, y una segunda parte, en la que los grupos se irán al rincón que les corresponda según la maestra haya indicado, cada vez que todos terminen la actividad de su rincón, se recogerán los materiales, e irán rotando de rincón en rincón hasta realizar las cuatro actividades de las distintas áreas de conocimiento, también hay que destacar que todas las actividades diseñadas para cada rincón, excepto el rincón de matemáticas, son actividades individuales a las que se acude en equipo. Las actividades correspondientes a cada rincón son las siguientes:

- Rincón de matemáticas: La actividad de este rincón se basa en el juego de mesa de la oca, pero en este caso sobre el caballito de mar, les explicaremos a los alumnos que al caballito de mar le gusta hacer carreras, y que cada uno tiene que coger su ficha (un caballito de mar), y por turnos cada miembro irá tirando los dados y contando casillas con su ficha. En el tablero hay un total de 30 casillas, ya que la finalidad principal de esta actividad es apreciar la seriación numérica del 1 al 30 y el conteo.

- Rincón de Lectoescritura: En este rincón los alumnos tendrán que poner en orden las letras de goma eva con velcros, que la maestra les dará a cada alumno, correspondientes al nombre de 'caballito de mar' estas letras irán en mayúscula, y para poner en orden las letras la maestra preparará un folio en A4 con las casillas, correspondientes a cada letra, en las que los niños tendrán que colocar cada letra en su casilla correspondiente, apoyándose en la visualización del nombre 'CABALLITO DE MAR' que la maestra habrá escrito en la pizarra. Cuando los alumnos hayan terminado de poner en orden las letras tendrán que escribir con lápiz debajo del nombre ordenado. 
- Rincón de la casita: La actividad de este rincón consiste en realizar dos puzles diferentes del caballito de mar, con el que los alumnos tendrán que diferenciar las diferentes partes del caballito de mar. Para ello la maestra primero les dará a los alumnos un folio en blanco y el dibujo de un caballito de mar dividido en 8 partes desordenadas, los alumnos tendrán que recortar estas piezas y pegarlas resolviendo el puzle, una vez terminen el primer puzle la maestra les repartirá el siguiente puzle de 6 piezas con el que tendrán que hacer lo mismo que con el anterior.

- Rincón de la plástica: En el rincón de plástica los alumnos deberán realizar, mediante la expresión plástica, su propio caballito de mar usando los materiales que hay en el rincón.

\section{FASE 3. APLICACIÓN Y CONSOLIDACIÓN DE LO APRENDIDO}

ACTIVIDAD 6: Érase una vez...

\begin{tabular}{|l|c|}
\hline \multicolumn{1}{|c|}{ Objetivo específico: } & Contenido específico: \\
\hline \multicolumn{1}{|c|}{ Reflexionar sobre la importancia del agua } \\
para el caballito de mar y sus compañeros, reflexionar \\
sobre las malas prácticas que se llevan a cabo en las \\
playas y que contaminan el agua, descubrir la \\
$\begin{array}{l}\text { importancia de no usar plásticos para reducir su } \\
\text { presencia en el mar. }\end{array}$
\end{tabular}

Temporalización: 40 minutos.

Descripción de la actividad:

Esta sesión se desarrollará en la zona de la asamblea, los alumnos se sentarán en círculo junto con la maestra, seguidamente la maestra colocará en el centro del círculo dos cartulinas tamaño A3, en ellas habrá dibujada una playa sin color y sin nada, encima de cada cartulina habrá un cartelito, en uno pondrá Villa Castañas y en la otra Villa Fantas. Después la maestra contará una historia, en la que los alumnos irán interviniendo por turnos, según lo indique la maestra, coloreando y colocando en las cartulinas los elementos que la maestra habrá preparado, que serán dibujos de botellas de plástico, peces coloridos, arena clara y arena oscura, colores oscuros y colores vivos...

Una vez acabada la historia, resultarían dos playas completamente diferentes, Villa Fantas contaminada con plásticos, coloreada de colores oscuros, con residuos sin personas y sin animales, y Villa Castañas, una playa colorida, con gente bañándose en el mar junto con los peces en el agua limpia y sin residuos.

Cuando la maestra acaba la narración, se pasará a una mini tertulia que la maestra guiará con una serie de preguntas sobre las dos playas resultantes, los alumnos observando el dibujo de las playas tendrán que llegar a una conclusión y reflejar mediante un dibujo su playa ideal, tras reflexionar con las siguientes preguntas: 
- ¿En cuál de las dos playas os gustaría estar?

- ¿En qué playa le diríais a vuestra familia que se bañase?

- ¿Cuál de las dos playas os parece más limpia?

- ¿Por qué?

- ¿A qué situación os recuerda esta historia?

- ¿Qué podemos aprender de la historia?

TALLER "La música del Mar"

\begin{tabular}{|c|c|}
\hline Objetivo específico: & Contenido específico: \\
\hline $\begin{array}{l}\text { Aprender el concepto de reutilización de } \\
\text { plásticos como actuación de reciclaje y ayuda al medio } \\
\text { ambiente. }\end{array}$ & $\begin{array}{l}\text { La reutilización de plásticos para dar le otro } \\
\text { uso que satisfaga una necesidad de la vida cotidiana. }\end{array}$ \\
\hline
\end{tabular}

En primer lugar, hay que destacar que esta actividad se llevará a cabo en dos partes, la parte explicativa en la asamblea y la parte de la puesta en práctica en las mesas de trabajo y de forma individual. En la primera parte la maestra explicará a los alumnos que muchas de las cosas que tiramos se pueden reutilizar, por ejemplo, las botellas de plástico se pueden utilizar para hacer instrumentos, por eso la maestra llevará 24 botellas que la encargada del comedor del colegio, en lugar de tirarlas, las habrá reservado a petición de la maestra. Seguidamente, la maestra les explicará que con las botellas van a realizar un instrumento y cómo realizarlo, la caña de agua.

Después de la explicación los alumnos se irán a sus mesas de trabajo para fabricar el instrumento que realizarán con: la botella de plástico, lentejas, algunas piedras, garbanzos y purpurina o estrellitas de colores para decorar, todo este material lo aportará la maestra y lo distribuirá por los grupos de trabajo junto con pinceles, pinturas y pegatinas para que los alumnos pinten por fuera la botella como quieran.

Cuando los alumnos hayan finalizado su instrumento, a modo de cierre, se realizará en la asamblea un concierto de cañas de agua que la maestra dirigirá marcando los ritmos e indicando que alumnos empiezan a tocar antes o después.

Cabe destacar que esta actividad está inspirada en el proyecto Garbage Music, que Shady Rabab, de Egipto diseñó, con su proyecto, enseñó a niños de zonas desfavorecidas a crear música con artículos de plástico para demostrar al mundo que podemos darle una nueva vida a los materiales que consideramos basura, por ello, ganó el premio Jóvenes Campeones de la Tierra del Programa de la ONU para el Medio Ambiente por aprovechar la oportunidad de sacar a los niños de las calles mientras ayudaba a reducir la contaminación por plásticos. 
TRABAJO COOPERATIVO: "Dónde había plástico, ahora hay un..."

\begin{tabular}{|c|c|}
\hline tivo específico: & Contenido específico: \\
\hline $\begin{array}{l}\text { Descubrir que la reducción del uso del } \\
\text { plástico ayuda a eliminar la contaminación de los } \\
\text { mares }\end{array}$ & $\begin{array}{l}\text { El plástico como material del que se puede } \\
\text { prescindir y utilizar otro en su lugar, que respeten el } \\
\text { entorno. }\end{array}$ \\
\hline
\end{tabular}

Temporalización: 45 minutos.

Descripción de la actividad:

Esta sesión busca cambiar hábitos de consumo como lo es el relacionado con el plástico, para ello, en primer lugar, la maestra en la asamblea explicará que lo que más ayuda al Mar menor y al planeta, es utilizar en lugar de plásticos otros materiales menos dañinos para el entorno, por ejemplo, en lugar de usar una botella de plástico podemos usar una de cristal. A continuación, la maestra explicará a los alumnos la problemática que deberán resolver con su grupo de trabajo.

Problemática: 'Muchos de los plásticos que usamos, a pesar de que los reciclamos acaban en el mar, aunque el reciclaje ayuda a que en el mar haya menos cantidad de plástico, no ayuda a hacer desaparecer el definitivamente todo el plástico del mar, por eso además del reciclaje y la reutilización, la mejor vía para ayudar al mar es usar en lugar de plásticos otro material, para así eliminar completamente los plásticos más dañinos'.

Tras lanzar la problemática, la maestra explicará la actividad en la asamblea, que consistirá en que los alumnos inventen una solución para una situación concreta, que la maestra explicará son dos y cada grupo tiene que proponer una solución para cada problema y dibujarla entre todos los miembros del grupo en un folio, las situaciones serán las siguientes:

- Es el cumpleaños de Pepe, y Pepe ha querido hacer una fiesta para invitar a sus amigos a merendar, la fiesta va a ser en el campo, porque a Pepe le gusta mucho el campo, pero en el cole ha aprendido que el plástico es mejor no usarlo mucho, por esto Pepe se preocupó porque iba a llevar vasos, platos y cubiertos de plástico, pero pensado en lo que ha dicho su maestra, ha decidido no llevar plásticos al campo, aunque tampoco quiere llevar vidrio porque tantos vasos y cubiertos pesan mucho, ¿Qué tipo de vasos, platos y cubiertos puede llevar Pepe al campo y que no pesen?

- El otro día que hizo tanto viento, la basura se salió de las papeleras del patio del colegio, y Roberta, la conserje del colegio, se ha encontrado muchos envoltorios de plástico, envoltorios de patatas, de chuches, de bocadillos, de chicles, bolsas de plástico... y se preocupó bastante, ¿Qué clase de envoltorios podemos usar para esas cosas, en lugar de usar envoltorios de plástico?

Los alumnos, en sus grupos de trabajo cooperativo, deberán elaborar una solución para cada una de las situaciones y posteriormente explicar a toda la clase las soluciones que han propuesto para los dos problemas. 
TRABAJO COOPERATIVO: "Ayudamos al Mar”

\begin{tabular}{|c|l|}
\hline Objetivo específico: & Contenido específico: \\
\hline Desarrollar habilidades de cooperación y \\
resolución ante la problemática ambiental del mar.
\end{tabular}

Temporalización: 50 minutos.

Descripción de la actividad:

En esta sesión se abordará principalmente la contaminación del Mar y su cuidado, para ello la primera parte de la sesión se llevará a cabo en la asamblea, los alumnos se sentarán en semicírculo mirando hacia la PDI ( Pizarra Digital Interactiva), la maestra les mostrará imágenes de Mares antes y después de ser contaminados y explicará que ha quedado de este modo por la contaminación del agua con residuos plásticos y basura que dejan los veraneantes, y por las aguas residuales que los agricultores desechan en el mar, por tanto, como consecuencia de que al haya tanta suciedad los rayos de sol no pueden llegar al fondo del mar y el agua no se puede renovar, es decir, limpiar.

Una vez explicado todo esto, la maestra les recordará a los alumnos que el caballito de mar nos pidió ayuda, y les preguntará qué se podría hacer para ayudar lo una vez lanzada esta problemática la maestra escuchará las soluciones que aportan los alumnos y mediante el diálogo en la asamblea encaminará sus aportaciones hasta la elaboración de distintos carteles con mensajes.

Estos carteles se elaborarán por equipos y cuando se finalicen se pegarán en el pasillo del centro escolar, para que así todo el colegio pueda aprender cómo cuidar el mar. Esta actividad está basada en el Aprendizaje Cooperativo, en la que los alumnos a partir de un problema tienen que elaborar una solución, esta es una metodología activa que contiene la clave del aprendizaje significativo.

Para la realización de esta actividad la maestra elaborará siete mensajes en la asamblea con la ayuda de los niños, que responderán a la pregunta de la maestra ¿¿Qué cosas podemos decirles a las personas para que ayuden a cuidar el mar menor?' y los mensajes que resultarán de sus respuestas con ayuda de la maestra si es necesario serán las siguientes:

- No tires plásticos a la playa.

- Cuando te estés bañando no tires basura al agua.

- El caballito de mar nos necesita.

- Si cuidamos el agua nos cuidamos a nosotros y a todos los animales.

- Si estás en la playa y ves algún residuo en la arena, tíralo a la papelera.

- No tires agua sucia al mar.

- Utiliza envases de vidrio o reutilizables.

- Evita usar cosas de plástico. 
Se harán seis equipos, cinco equipos formados por cuatro alumnos y un equipo formado por cinco alumnos, cada equipo escogerá un mensaje, esto se hará por sorteo con la ayuda de la maestra. Tras elegir el mensaje deberán de ponerse de acuerdo los miembros del grupo para hacer entre todos un dibujo en una cartulina A3 que explique lo que dice su mensaje, una vez que hayan terminado, la maestra pegará en la parte de arriba del dibujo el mensaje escrito e irán todos a pegar sus carteles en el pasillo de su aula.

\section{FASE 4. CIERRE Y EVALUACIÓN}

Taller: 'Hemos descubierto que...'

\begin{tabular}{|c|l|}
\hline Objetivo específico: & Contenido específico: \\
\hline Valorar lo que se ha aprendido a lo largo del & Todo lo aprendido a lo largo del proyecto. \\
proyecto. & \\
\hline
\end{tabular}

Temporalización: 40 minutos.

Descripción de la actividad:

En esta sesión los alumnos deberán reflexionar sobre todo lo que han aprendido, será una sesión de cierre en la que los alumnos deberán explicar por grupos, los cuatro grupos de trabajo, lo que han aprendido del mar menor y del caballito de mar, para ello la maestra les dejará tener una pequeña reunión con su grupo para acordar lo que va a decir cada miembro del grupo y para hacer un dibujo que apoye su explicación en la cartulina que la maestra le dará a cada equipo.

Cuando haya pasado el tiempo límite la maestra irá nombrando al grupo que le toca exponer lo que ha aprendido hasta que todos los grupos hayan salido, esta actividad junto con las producciones hechas por los alumnos serán el recurso de la maestra para evaluar los objetivos conseguidos.

\section{CONCLUSIÓN}

El presente proyecto pretende ser una aportación una modesta aportación que ayude a mejorar la educación para la sostenibilidad en las aulas de infantil. Aborda una de las problemáticas ambientales de la actualidad. Se trata de dar a conocer a los niños la conexión directa que hay entre las personas y su entorno; además, trata manera práctica la educación para el desarrollo sostenible, en la etapa de infantil, mediante metodologías que se adaptan al alumnado y lo ponen en el centro del proceso de enseñanzaaprendizaje, haciéndolo agente activo del mismo.

Con este proyecto estaríamos contribuyendo a cumplir con los objetivos de la Agenda 2030 de la ONU, concretamente con el objetivo se estaría abarcando el objetivo catorce, al que compete "la vida submarina” y, que tiene como finalidad: "Conservar y utilizar en forma sostenible los océanos, los mares y los recursos marinos para el desarrollo sostenible”. Para esta finalidad, la UNESCO desarrolló una serie de objetivos de aprendizaje y distintos temas con los que abordar sostenibilidad de la vida submarina. El 
presente trabajo pretende ser una ayuda para conseguir siguientes objetivos de aprendizaje propuestos por la UNESCO (2017, p. 38):

De los objetivos de aprendizaje cognitivos:

- "El/la alumno/a comprende el vínculo que mucha gente tiene con el mar y la vida que sustenta, incluyendo el rol del mar como fuente de alimento, trabajo y oportunidades" (objetivo número 2)

De los objetivos de aprendizaje socioemocionales:

- "E1/la alumno/a es capaz de demostrar a las personas el impacto del hombre sobre los océanos (pérdida de biomasa, acidificación, contaminación, etc.) y la importancia de océanos saludables y limpios." (objetivo número 2)

Y el tema de este proyecto, que también aparece en el documento de la UNESCO:

- "Contaminantes del océano: plásticos, microperlas, aguas residuales, nutrientes y químicos” (p. 39)

Hacer partícipes a los más pequeños de esta grave responsabilidad humana se convierte en una herramienta eficaz para promover el desarrollo sostenible, pues además de preparar a las futuras generaciones de adultos que tendrán que tomar decisiones, les involucra directamente desde su infancia a ellos y a sus familiares.

$\mathrm{Y}$ un punto que hace interesante nuestro proyecto radica en que se podrá llevar a cabo, de forma generalizada, en cualquier aula, con una sencilla adaptación de objetivos y contenidos, en orden a la edad a la que vaya dirigido. 


\section{BIBLIOGRAFÍA}

Beresaluce, R. (2009). Las escuelas municipales de Reggio Emilia como modelos de calidad en la etapa de Educación Infantil. Editorial Club Universitario.

Candelo, C., Ortiz, G., \& Unger, B. (2003). Hacer talleres. Una guía práctica para capacitadores, Colombia, Fondo Mundial para la Naturaleza (WWF), In went (DSE), Instituto para la Comunicación en Organizaciones.

Careaga, A., Sica, R., Cirillo, A., \& Da Luz, S. (2006). Aportes para diseñar e implementar un taller. Comunicación presentada en el 8vo. Seminario-Taller en Desarrollo Profesional Médico Continuo (DPMC). 2 das Jornadas de Experiencias educativas en DPMC. Departamento de Maldonado, Uruguay. Delors, J. (1996). Los cuatro pilares de la educación. En J. Delors, La educación encierra un tesoro. (pp. 91-103). Madrid: Santillana, Ediciones UNESCO.

Domínguez, G. (2003). En busca de una escuela posible. Revista Interuniversitaria de Formación del Profesorado, 17 (3), 29-47.

García-Ruíz, R. (2013). Enseñar y aprender en Educación Infantil a través de proyectos. Editorial de la Universidad de Cantabria.

Miralles, P. y Rivero, A., (2012). Propuestas de innovación para la enseñanza de la historia en Educación Infantil. REIFOP, 15(1), 81-91.

Johnson, D.W, Johnson, R.T. y Holubec, E. J. (1999). El aprendizaje cooperativo en el aula. Buenos Aires: Paidós.

Laguía, M. J. y Vidal, C. (2000). Rincones de actividad en la escuela infantil (0 a 6 años). Barcelona: GRAÓ.

Vizcaíno, I. M. (2008). Guía fácil para programar en educación infantil. Trabajar por proyectos. Madrid: Wolters Kluwer España.

Directrices y recomendaciones encaminadas a reorientar la formación de docentes para abordar el tema de la sostenibilidad La educación para el desarrollo sostenible en la práctica Documento técnico $\mathrm{N}^{\circ} 2$ 2005Sector de Educación de la UNESCO Decenio de las Naciones Unidas de la Educación para el Desarrollo Sostenible (2005-2014) Organización de las Naciones Unidas para la Educación, la Ciencia y la Cultura

UNESCO. (2017). Educación para los Objetivos de Desarrollo Sostenible. Objetivos de aprendizaje. Recuperado de URL: https://web.unican.es/unidades/igualdad/SiteAssets/guia-derecursos/responsabilidad-social-universitaria/EdS_ODS.pdf 